\title{
Cross Layered MAC Design for RF Energy Harvesting Sensor Network
}

\author{
Thangavelu Sasikala1, Veerasamy Jawahar Senthil Kumar² \\ Department of ECE, College of Engineering, Anna University, Chennai, India \\ Email: sasi1504@gmail.com
}

Received 1 May 2016; accepted 20 May 2016; published 28 July 2016

Copyright (C) 2016 by authors and Scientific Research Publishing Inc.

This work is licensed under the Creative Commons Attribution International License (CC BY). http://creativecommons.org/licenses/by/4.0/

c) (i) Open Access

\section{Abstract}

The main research objective in wireless sensor networks (WSN) domain is to develop algorithms and protocols to ensure minimal energy consumption with maximum network lifetime. In this paper, we propose a novel design for energy harvesting sensor node and cross-layered MAC protocol using three adjacent layers (Physical, MAC and Network) to economize energy for WSN. The basic idea behind our protocol is to re-energize the neighboring nodes using the radio frequency (RF) energy transmitted by the active nodes. This can be achieved by designing new energy harvesting sensor node and redesigning the MAC protocol. The results show that the proposed cross layer CL_EHSN improves the life time of the WSN by $40 \%$.

\section{Keywords}

\section{Wireless Sensor Network, Cross Layer Design, RF Energy Harvesting, Lifetime Enhancement}

\section{Introduction}

In recent years, wireless sensor networks (WSNs) have gained worldwide attention for use in different applications such as military target tracking and surveillance, natural disaster relief, biomedical health monitoring, and industrial automation. Sensor nodes are spatially distributed across a large area of interest and they are capable to sense, measure, gather information and transmit the data to the user. They are small in size, inexpensive, and can be deployed in large numbers. The nodes are typically equipped with radio transceivers, micro-controllers, and batteries. They are deployed for applications where recharging of the batteries is not possible. Hence, the limited energy creates serious problems in the network operation.

Sensor nodes are constrained in energy supply and bandwidth. Thus, innovative techniques to eliminate energy inefficiencies which will shorten the lifetime of the network are highly required. Such constraints combined with a typical deployment of large number of sensor nodes pose many challenges to the design and management 
of WSNs and therefore necessitate energy-awareness at all layers of the networking protocol stack.

Recent advances in the area of wireless energy transfer [1]-[3] allow sensors to recharge during network operation, thereby extending their lifetimes and minimizing application downtime.

In this research, we propose a cross layer based energy harvesting sensor node and novel MAC protocol design for recharging the sensor nodes during communication. Here we redesign the sensor node with energy harvesting module which consisting of specially designed antenna to convert the RF signal into an electrical signal with associated circuits and a controller switch. Based on the control signal obtained as output from the cross layered MAC protocol the switch will connect the antenna to transmit mode or harvest mode.

\section{Related Work}

\subsection{Design of Energy Harvesting Sensor Node}

Most sensor nodes used in WSNs today rely on a limited energy source like primary batteries to operate. One attempt [1] to solve the energy problem is to make use of some mobile sensor nodes to deliver energy to other sensor nodes. Another solution that has been adopted is to make use of sensor nodes that rely on energy harvesting devices [2] for power. Some examples of sensor nodes using energy harvesters have been deployed in test beds. For example, in [3], solar-powered sensor nodes are used to evaluate robust multi-target tracking algorithms. Other solar-powered sensor network test beds are illustrated in [4] and [5]. The power harvesting devices provides very good solution [6] for this problem. Energy harvesting wireless sensors has also been developed for monitoring the structures of aircraft [7]. The recent research on powering Mica2 sensor motes by harvesting the energy contained in radio (RF) electromagnetic waves in [8] indicated the potential for large scale deployment of this technology.

To ensure that energy is not unnecessarily lost during the transfer from the harvester to the wireless sensor, a low-power maximum power point tracker (MPPT) circuit [9] has been proposed to efficiently transfer the harvested solar energy to rechargeable batteries even in non-optimal weather conditions. In [10], a vibration-based harvesting micro power generator is used to scavenge environmental vibrations for use in a sensor node. Traffic sensors can also be solely powered by the short duration vibrations when a vehicle passes over the sensor [11]. Devices with direct contact to the human body can harvest the energy radiated from the human body by means of thermo generators (TEGs) [12]. To address the needs of telecommunications and other embedded applications, design of micro structured thermoelectric devices has been proposed in [13]. There are also commercially available sensor nodes developed by Microstrain [14] which rely on ambient energy harvesting for power. In [15] harvested and used energies are from two sources, viz. solar and mechanical energy. A Solar Energy harvesting development kit, produced by Texas Instruments [16], cerametrics [17] produces vibration-based energy harvesters that can replace batteries and can used to power wireless sensor nodes. Details on other commercially available energy harvesters for sensor nodes are available in [18]. Energy harvesting powered sensor nodes for specific applications, like indoor climatic monitoring and industrial environmental control, are commercially available. Battery-less sensor nodes that can be powered by any energy harvester are produced by Ambiosystems [19]. Another company, EnOcean [20] produces sensing systems that can power themselves by harvesting ambient energy from the environment.

\subsection{MAC Protocols for Energy Harvesting}

In [21] the authors propose a survey of MAC protocols for M2M communication in mHealth application. It is found that the author fail to discuss about the RF energy harvesting capability of the nodes and use of this harvested energy in a cross layered approach to design the MAC. The authors in [22] designed a co-operative energy harvesting MAC protocol for WBAN applications. Their proposed protocol operates only for co-operative mode of scenarios. Where they use relays that complicates the system design. In [23], the authors propose information exchange in large scale networks with wireless energy harvester and dynamic power splitting. This work reports data exchange using co-operative communication in WSN and derives the throughput for direct communication scenario and relayed scenario. Their findings highlight that direct communication performs better than the co-operative scenario.

The authors in their work [24] reported MAC protocol that combines polling and probabilistic contention access for wireless body area network. [25] proposes network coding aided co-operative communication for 
large scale network, where the relays are able to harvest energy emitted by wireless transmissions. They derive the probability of successful data exchange and network lifetime gain. They did not design a MAC protocol for data exchange.

The authors in [26] propose a joint power QoS control scheme that has three modules that interact with each other to make optimal use of energy and achieve QoS for WBAN application. In this work the energy is harvested in the human environment.

The main contributions of this research work are to 1) Design a RF energy harvesting sensor node for an operating frequency of $915 \mathrm{MHz}$ and evaluate its harvesting capability in real time wireless environment; 2) To design a cross layered MAC protocol that controls the operation of the node to be in harvest mode and at active mode. Theoritical equations are written to decide on the energy harvest time based on IEEE 802.15.4 protocol; 3) A novel energy request packet transmission is introduced to harvest energy from neighboring nodes to enhance the throughput of the network.

The rest of the paper is organized as follows: Section 3 discusses the proposed energy harvesting sensor node, Section 4 presents the details of network using the designed energy harvesting sensor node, and Section 5 discusses the medium access design for the CL_EHSN network. The performance evaluation and results are presented in Section 6, and finally Section 7 concludes the paper with future work.

\section{The Sensor Node with RF Energy Harvesting Capability}

The proposed energy harvesting sensor node (EHSN) shown in Figure 1 has three main modules: the traditional WSN node modules such as Sensors, Processor, Memory and Radio module. All these modules are powered by an internal rechargeable battery. The second module is the RF energy harvest (EH) module which contains matching and rectifying circuits. This converts the RF energy impinging on the antenna to variable current. This variable current is further rectified, filtered and stored in the battery. The third module is the switch that toggles between the transceiver module and the energy harvesting module based on the harvesting time $\left(t_{H}\right)$ and active time period defined by the MAC protocol. The antenna is connected to the Tx/Rx for transmission and reception of data during the active time period of the node, and for the rest of the time the switch connects the antenna to the EH module, enabling the node to recharge.

\section{EHSN Network}

The wireless sensor network created using the energy harvesting sensor nodes (EHSN) is given in Figure 2. The nodes are deployed randomly in the region of interest. In this proposed work, we employ flat topology, that is, all nodes are equal in all respect. Here we exploit the property of the antenna to convert electromagnetic energy to electrical signal, which is further converted to DC and stored in the battery. As every node in WSN has an antenna for data transmission and reception, we use the same antenna for energy harvesting purpose with novel design in sensor node and MAC protocol to achieve this goal. The proposed MAC protocol decides when a node should transmit and when to charge.

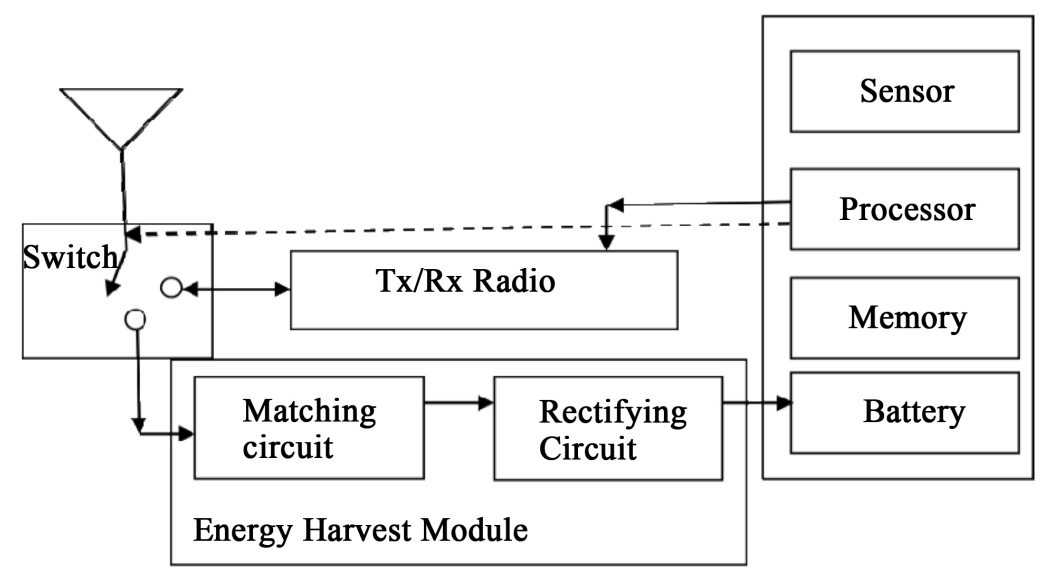

Figure 1. The energy harvesting sensor node (EHSN). 


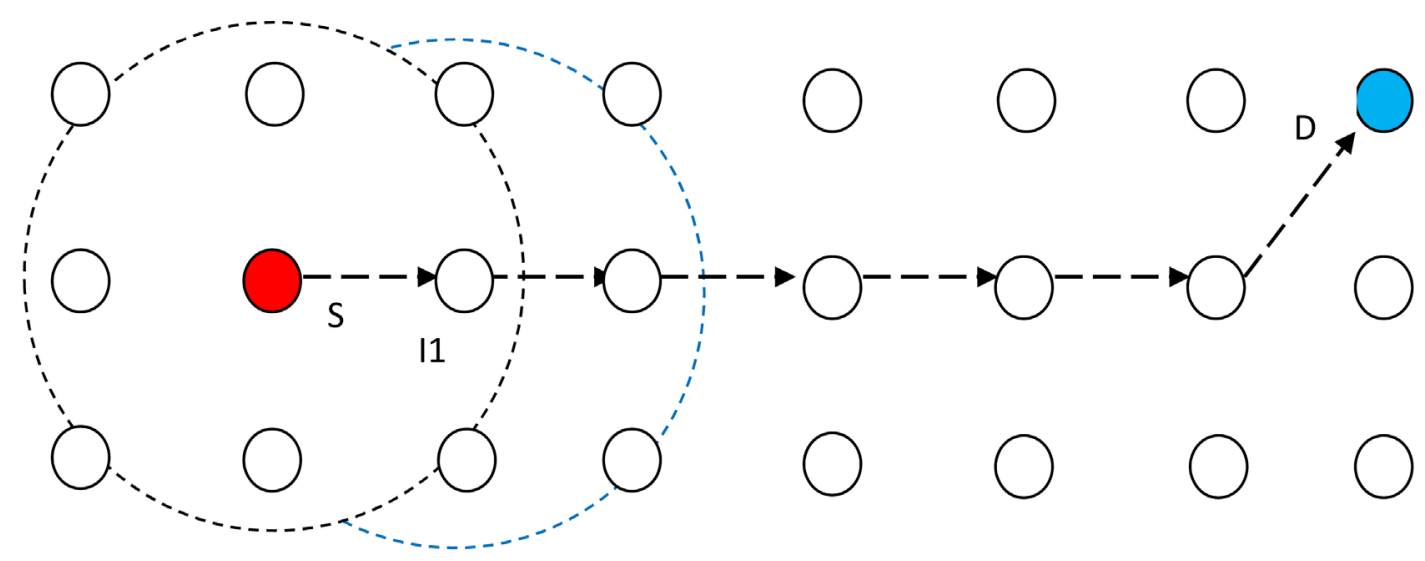

Figure 2. The energy harvesting sensor network.

\section{Cross-Layered MAC Design for EHSN (CL_EHSN)}

Most of the commercially available sensor nodes use Zigbee radio, which uses IEEE 802.15.4 MAC. This MAC does not support rechargeable networks. Hence, we propose new cross layered MAC protocol for the proposed EHSN network as illustrated using Figure 3.

The node that has data to transfer to the sink finds a path to the sink using well known routing protocols. The path discovered by the route discovery phase is passed to MAC to identify the next hop nodes. The nodes along the routing path are involved in data transmission. The MAC protocol provides access to these nodes to transfer data link by link. The source node transmits the data to the next node along the routing path. As illustrated in Figure 2, the source node S transmits RTS packet to the next node I1. This signal will alert all the neighbors of source node S to be ready to get charged. The receiving node I1 transmits clear to send (CTS) to S. This enables all the neighboring nodes of I1 to be alert to get charged. The length of the data frame mentioned in the RTS and CTS decides charging time of the neighbors as given by Equation (1)

$$
\mathrm{T}_{\text {cns } 1}=n / \mathrm{R}
$$

where, $\mathrm{T}_{\text {cns } 1}$ is the charging time of the neighboring nodes of $\mathrm{S}$ for one cycle, $n$ is the total number of bits in one MAC frame and $\mathrm{R}$ is the rate of transmission. The charging time $\mathrm{T}_{\text {cns } 1}$ is equal to the transmission time of the source node $\mathrm{T}_{\text {stx. }}$.

Figure 4 shows the charging time for an energy harvesting node in one super frame.

For the mote that operates at RF frequency $915 \mathrm{MHz}$, with data transmission rate $\mathrm{R}$ of $250 \mathrm{Kbps}$, to deliver a frame of 1000 bits $T_{s t x}=n / R=4$ ms. One super frame in 802.15.4 is of length $15 \mathrm{~ms}$. Assuming that a node has only one guaranteed time slot (GTS) in one super frame, the total charging time for an active node from any surrounding transmission will be the difference between one super frame time $\mathrm{T}_{s f}$ and $\mathrm{T}_{s t x}$, which is about $11 \mathrm{~ms}$. If the node is not involved in the data transmission or reception, the node can charge for the entire super frame time $\mathrm{T}_{s f}$ at $15 \mathrm{~ms}$.

The node is programmed to operate in two different modes known as active mode and harvest mode, as shown in Figure 5.

The duration of active mode and harvest mode is adaptive. If the remaining battery power is greater than the $\mathrm{B}_{\mathrm{th}}$ threshold, the operation is similar to that of a normal mote. The duration of the active period depends on the duty cycle defined by the MAC. During the active period the node will transmit and receive the data. During the harvesting period, the antenna is connected to the EH circuit as shown in Figure 6. The RF signal transmitted by the neighboring nodes is converted to energy and stored in the battery.

\subsection{MAC Operation}

The operation of MAC is explained as follows. Depending on the type of received packets the node will perform various operations. If it is in active time then the node will check for the availability of data. If the data to be transmitted are ready, the controller puts the switch in the transmitting mode and enables the transmission chain circuitry. If the battery power $\mathrm{B}_{\mathrm{p}}$ is greater than the threshold $\mathrm{B}_{\mathrm{th}}$, a ready to send (RTS) is transmitted and RTS 
Network layer(Route)

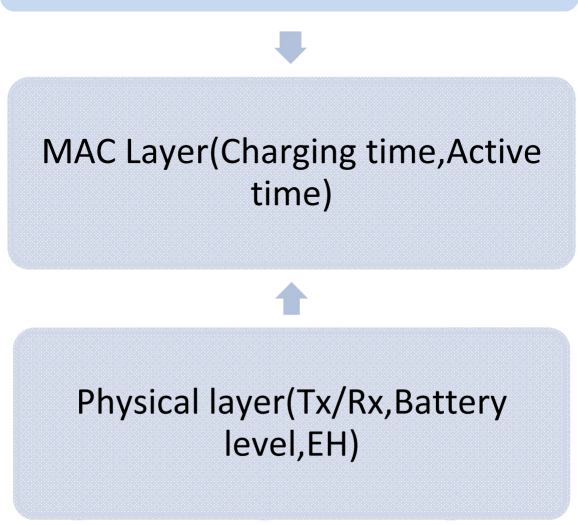

Figure 3. The cross layer parameters.

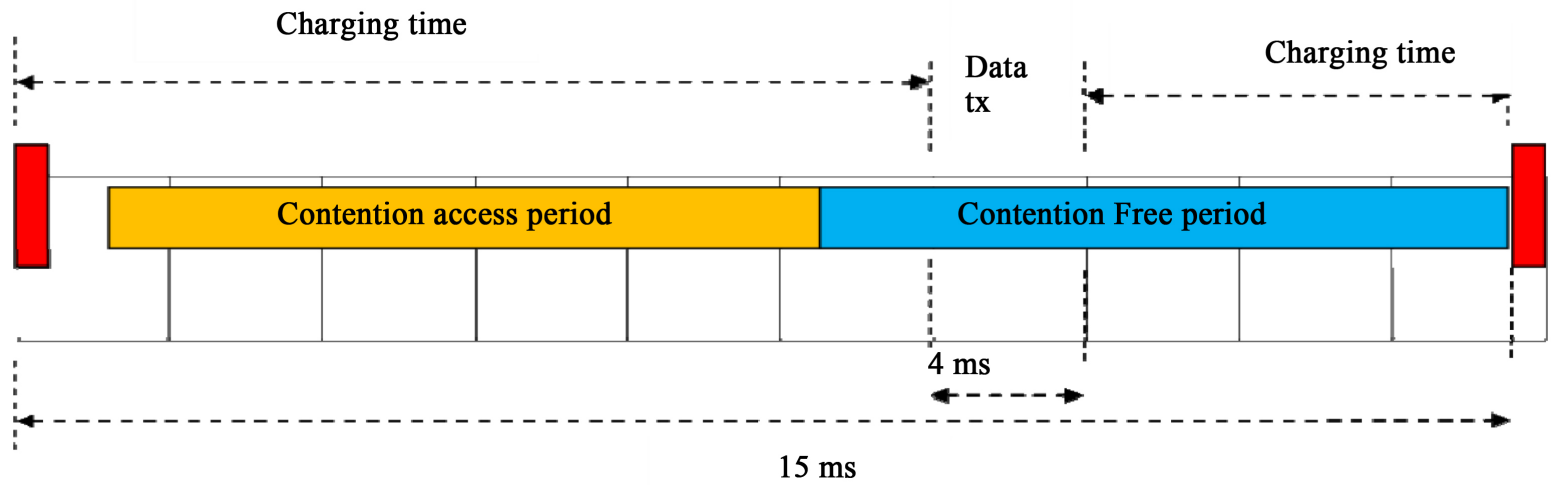

Figure 4. The charging time for an EHSN in one super frame.

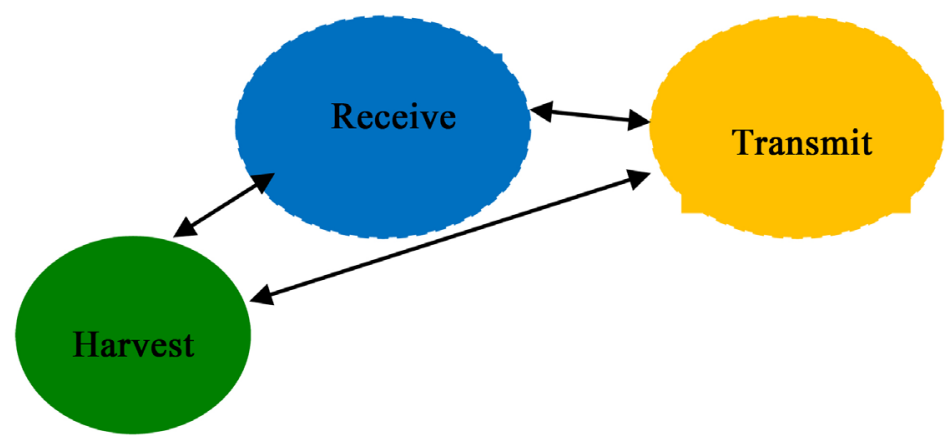

Figure 5. MAC state diagram.

timer is started. If RTS timer ends before clear to send (CTS) packets are received, the data is converted to RF signals and transmitted. If no CTS is received before RTS timer ends, the retry counter is incremented and the mote once again transmits RTS till the retry counter reaches its maximum predefined limit (maxRetry).

If the $B_{p}$ is less than or equal to $B_{t h}$, the node will not be allowed to transmit data. The active time period is abruptly cut down and the node is moved to harvest mode. The node will be in harvest mode till its $B_{p}$ is greater than $\mathrm{B}_{\mathrm{th}}$. Once the node reaches the required power level from the transmissions around, then it resumes the 
normal operation. Even after few cycles if the $B_{p}$ is not resumed then the node will transmit energy request packets.

There are five different packets are used in the protocol namely data packets, RTS, CTS, Energy request packets and Energy packets. If the received packet is RTS the node will respond with CTS and wait for data packets. If the energy required packet is received, the node prepares energy packets and transmits it. The whole operation of the MAC layer which is proposed in this paper is given in the following signal flow diagram. The MAC flow diagram for packet transmission and packet reception are shown in Figure 6 and Figure 7.

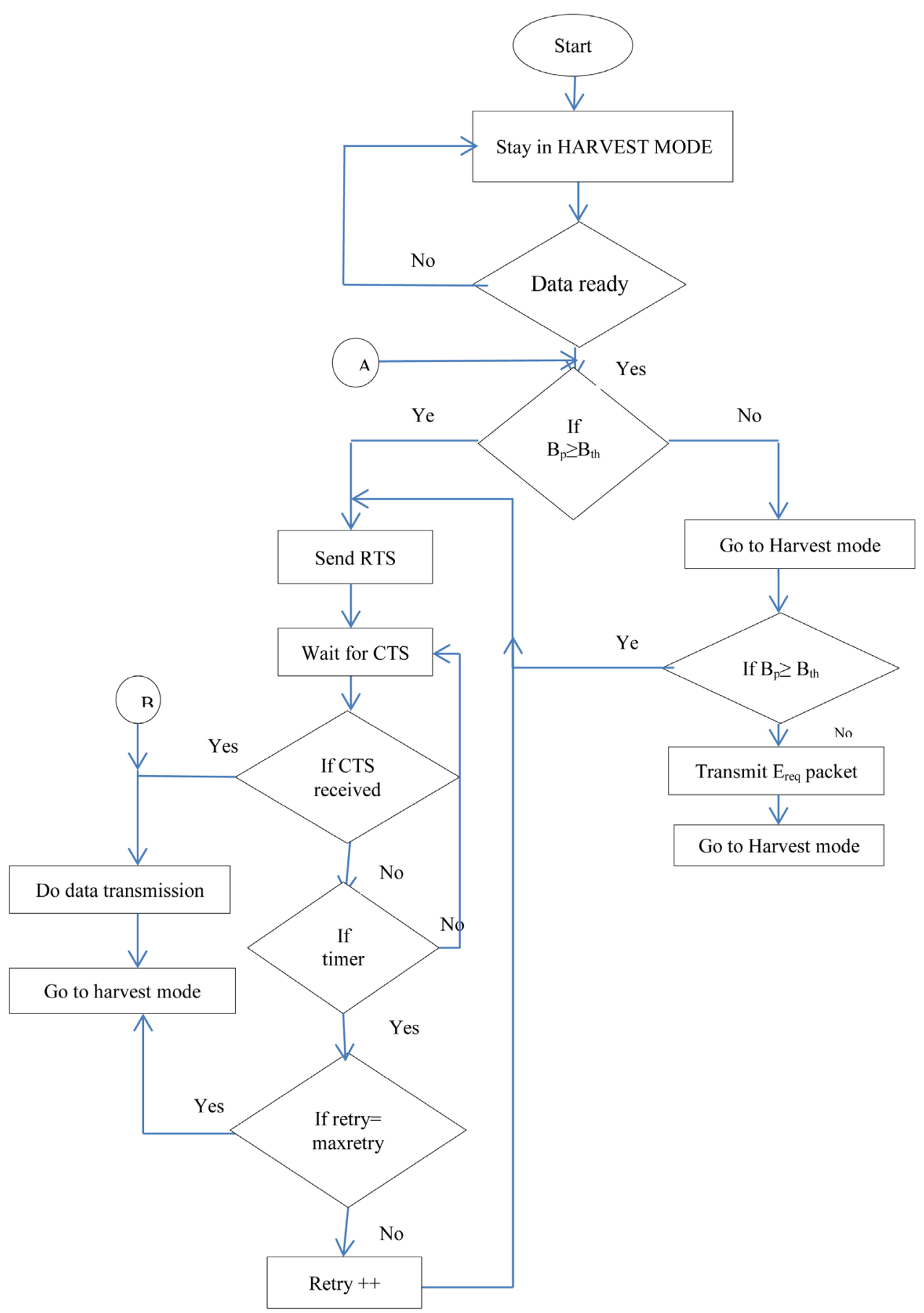

Figure 6. MAC flow diagram for packet transmission. 


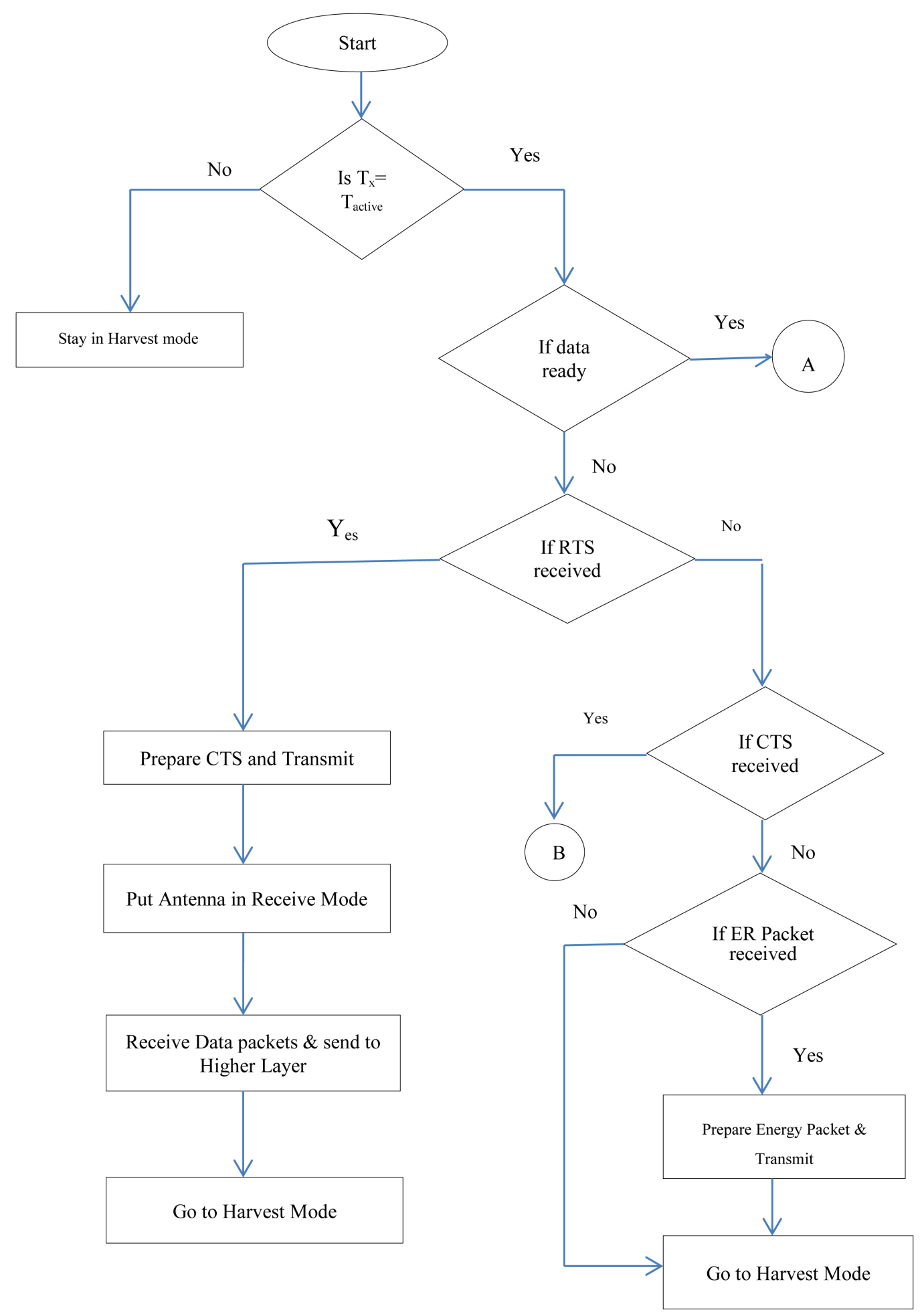

Figure 7. MAC flow diagram for packet reception.

\subsection{Algorithm}

\subsubsection{Packet Transmission Algorithm}

Step 1: Initially the node is in the harvest mode. 
Step 2: When the data is ready the node will check for the battery power $\left(B_{p}\right)$.

Step 3: If the battery power is greater than the fixed threshold value $\left(\mathrm{B}_{\mathrm{th}}\right)$, it will send the RTS and set the RTS timer and wait for the CTS packet.

Step 4: If CTS received before RTS timer ends, it will do data transmission. Otherwise it will increment the Retry counter.

Step 5: Repeat step 3 and 4 until retry counter reaches maxRetry.

Step 6: If the remaining battery power is less than the threshold value, the node will abort the active mode, and enters into the harvest mode.

Step 7: If the remaining battery power is not greater than the threshold value after two super frame periods, the node will transmits the energy request packet and enters into the harvest mode.

\subsubsection{Packet Reception Algorithm}

Step 1: If the present time is active period for the node, it will check for the availability of the data.

Step 2: If the data is ready it will do data transmission as per the section 5.2.1. Otherwise it will go to harvest mode.

Step 3: If the received packet is RTS, the node will send the CTS, and put the antenna in the receive mode. Once the reception is over, the received data are transferred to the next higher layer, and then the node will enter into the harvest mode.

Step 4: If the received packet is not a RTS packet, then the node will check whether the received packet is CTS .

Step 5: If the received packet is CTS, then the node will go to data transmission phase.

Step 6: If the received packet is not a CTS, then it will check whether the received packet is energy requests packet.

Step 7: If the received packet is energy request packet, then the node will prepare the energy packets and transmit it. Otherwise it will go to harvest mode.

\subsection{Rate of Harvesting Being Less than Rate of Usage}

Due to continued transmissions and receptions, there is a probability that a node's remaining battery power may reach an alarming level as the rate of usage is always greater than rate of harvesting. When the battery power level reaches a threshold $B_{t h}$, the MAC first hands over the channel to any of its neighboring nodes (differ its transmission). If a neighbor node grabs the channel for its transmission then the hungry sensor node can harvest energy. If after an interval of time, none of its neighbor is active to take up the channel then the MAC prompts the routing layer to transmit energy request packet. The neighboring nodes on receiving this request prepare energy packets and transmit in order to boost up the requesting node energy level. For simplicity, it is assumed that every neighbor whose battery power level is greater than threshold will definitely prepare an energy packet and transmit. Therefore, one energy request triggers $\mathrm{N}$ energy packet transmission; where, $\mathrm{N}$ is the number of live neighbors to a node. If a node does not get any reply to its energy request packet, this implies all its neighbors are dead or it is an orphan node.

\section{Performance Result and Discussions}

An EHSN node is designed as shown in Figure 8. A micro strip patch antenna operating at $915 \mathrm{MHz}$ is designed using Computer Simulation Tool (CST) software.

The EH circuit with matching network and rectifier circuit is designed and fabricated. For experimental purpose, one antenna is connected to RF energy source and excited at various power levels. The received signal power is measured and given in Table 1.

The transmitted power of the antenna is fixed to $15 \mathrm{dbm}$, and the distance between the transmitting and receiving node is varied. The variation in the received power is tabulated in Table 2.

The results from Table 2 show that as the excitation power increases, more power is harvested; and the harvested power decreases as the distance between the nodes increases.

This energy harvesting circuit is connected to various commercially available motes like MICA, MICA2 and Telos-B to convert the normal mote to energy harvesting mote. The power consumed by the nodes during the active period is given in Figure 9. Figure 10 shows the energy harvested of various nodes for the harvest period 


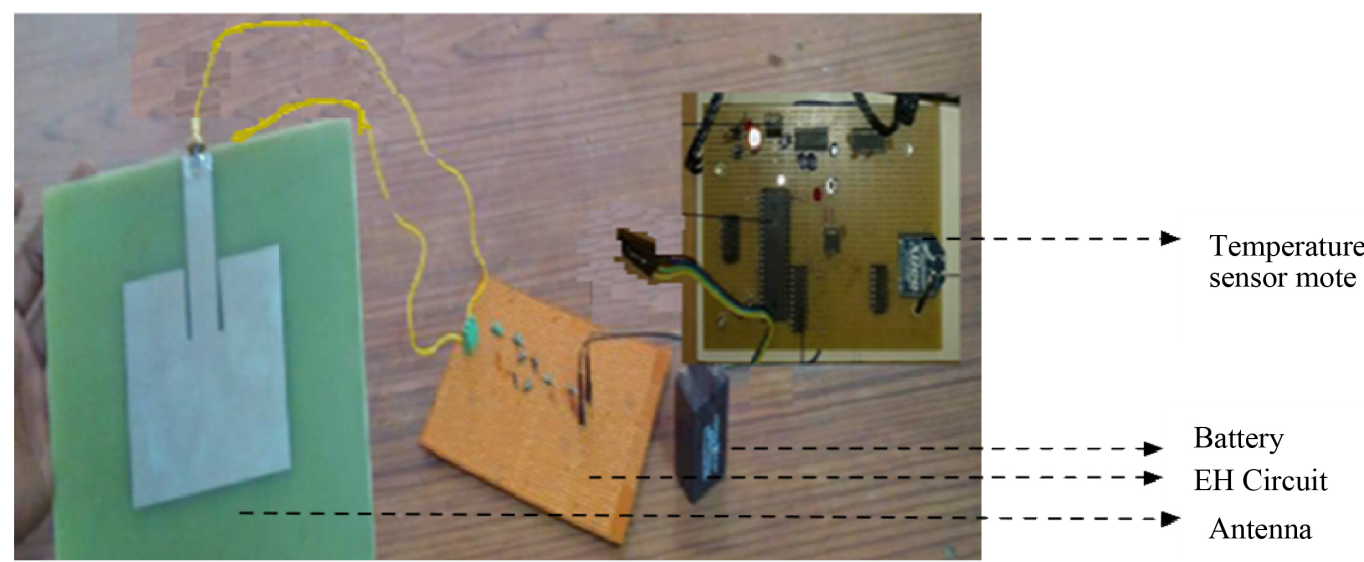

Figure 8. Energy harvesting sensor node.

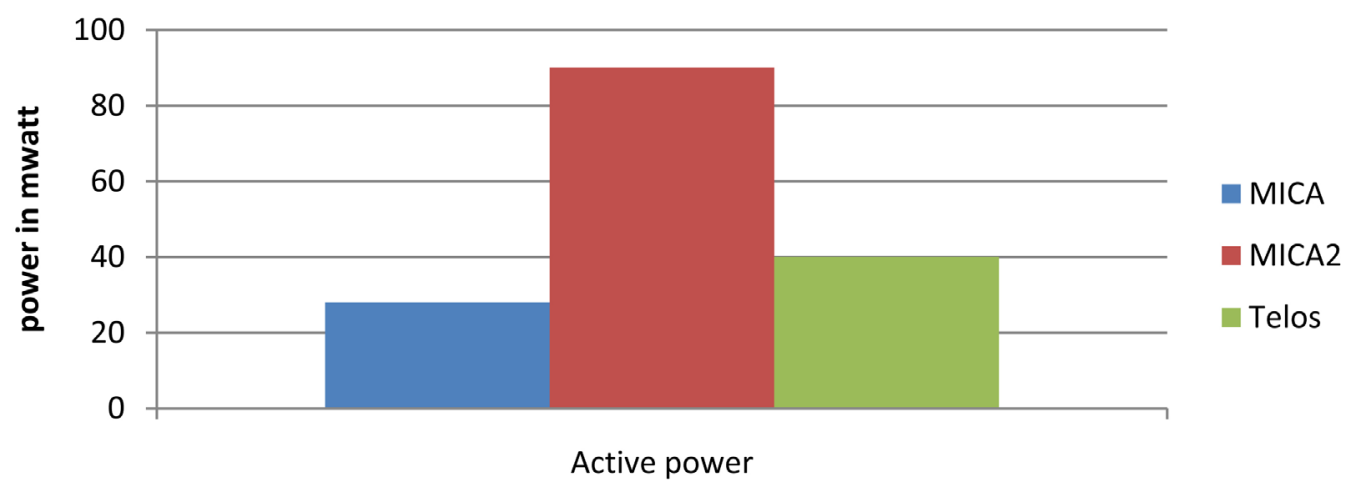

Figure 9. Energy consumption of different motes during active period.

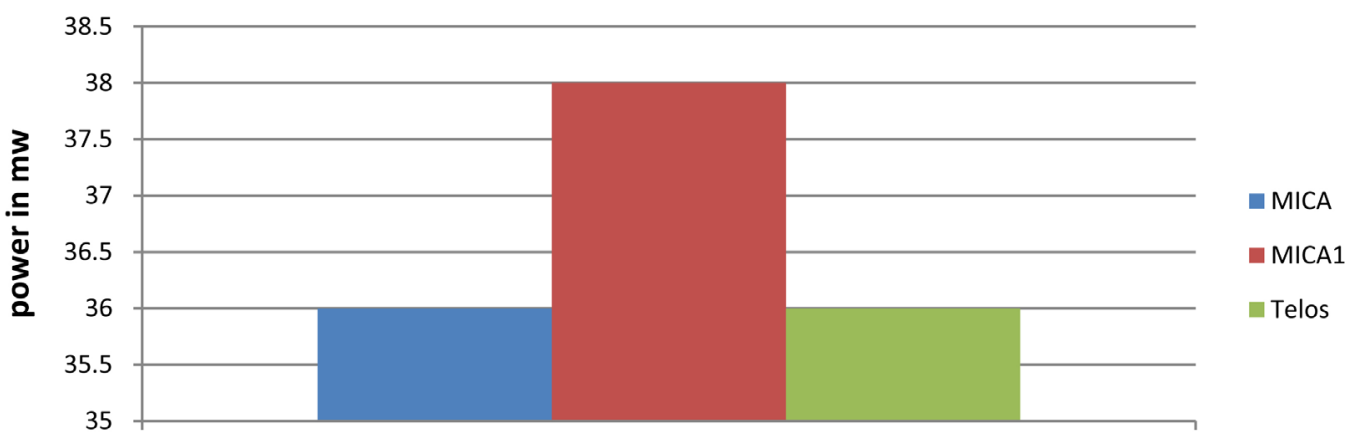

$\mathrm{EH}$ in $11 \mathrm{~ms}$

Figure 10. Energy harvested by different motes with EH for the harvest period of $11 \mathrm{~ms}$.

of $11 \mathrm{~ms}$.

The amount of power required for MICA mote in active mode is about $27 \mathrm{~mW}$, for MICA2 it is about $89 \mathrm{~mW}$, and for Telos-B it is about $41 \mathrm{~mW}$. The EH circuits connected to this mote are enabled and examined for $11 \mathrm{mS}$. The power harvested by the motes is about $36 \mathrm{~mW}$ when powered by $15 \mathrm{dbm}$ transmitted signal at a distance of $15 \mathrm{~cm}$.

Figure 11 shows the lifetime enhancement achieved by the network when operated with EH circuit.

From the result it is found that the total lifetime is enhanced by $43 \%$ for MICA motes compared to that of without EH circuit. For MICA2, the enhancement is less because the power spent during active mode is high. For Telos-B the amount of energy harvested is about $40 \%$. 
Table 1. Energy harvested as transmitting power increases (distance $=15 \mathrm{~cm})$.

\begin{tabular}{cccc}
\hline Transmitted Power (dbm) & Received Power (dbm) & Received Power (mw) & Output of EH Circuit (mv) \\
\hline-5 & -43 & $5.011 \times 10^{-5}$ & 11 \\
0 & -38 & $1.585 \times 10^{-4}$ & 11.5 \\
5 & -33 & $5.011 \times 10^{-4}$ & 12.3 \\
10 & -28 & $1.5848 \times 10^{-3}$ & 13.5 \\
15.5 & -18 & $1.5 \times 10^{-2}$ & 16 \\
16.2 & -12 & $6.3 \times 10-2$ & 17.2 \\
\hline
\end{tabular}

Table 2. Energy harvested as distance between the sensor increases (transmitting power $=15, \mathrm{dbm}=36 \mathrm{mw}$ ).

\begin{tabular}{cccc}
\hline Distance between the tx and rx node $(\mathrm{cm})$ & Received power $(\mathrm{dbm})$ & Received power node $(\mathrm{mw})$ & Output of EH circuit (mv) \\
\hline 15 & -18 & $1.5 \times 10^{-2}$ & 15 \\
20 & -24.2 & $3.8 \times 10^{-3}$ & 10 \\
25 & -29 & $1.25 \times 10^{-3}$ & 7.7 \\
\hline
\end{tabular}

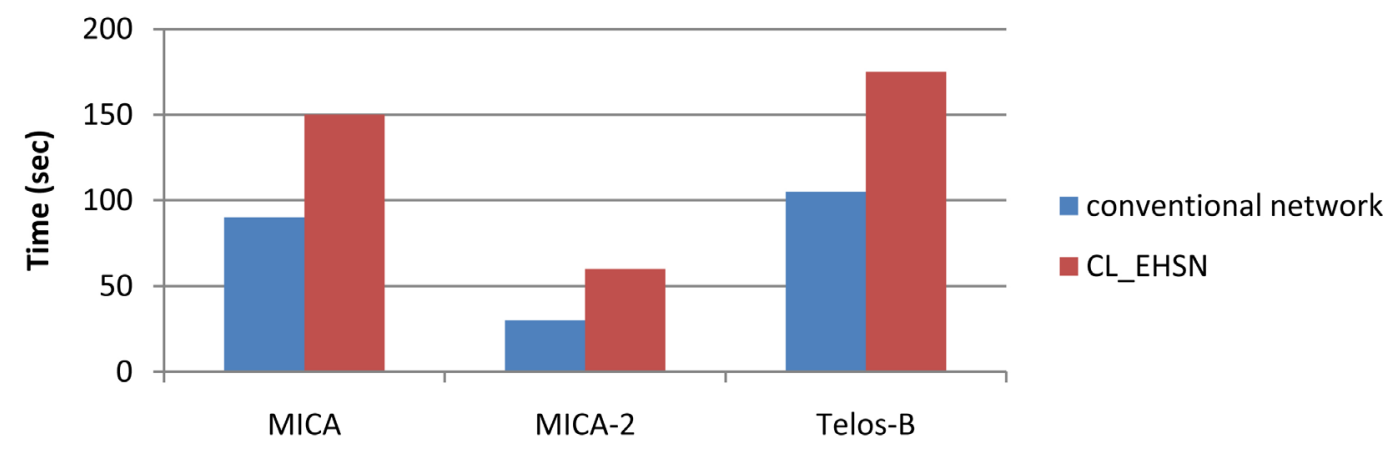

Figure 11. Lifetime enhancement comparison.

The average network throughput is evaluated as simulation time is increased as shown in Figure 12. From the result it is observed that as the simulation time increased the throughput of the network for the conventional network is decreased. This is because of energy depleted nodes increases as time increases. The proposed algorithm CL_EHSN achieves an overall improved performance compared to the conventional network. This is because of the energy harvesting nature of the network.

The number of nodes in the network is increased and its effect on average throughput of the network is given in Figure 13. When the number of node increases, the throughput decreases for the conventional network. This is because as the number of nodes increases, the routing path involves more nodes to deliver the packet to the sink. Hence the energy of the network decreases which leads to lesser throughput of conventional network. Whereas the proposed CL_EHSN harvest energy from its neighbors and keeps up the throughput by $15 \%$.

The performance of the average delay with increased number of nodes in the network is given in Figure 14 . When the number of node increases, uses more number of hop counts for the packet transmission. More number of hop counts results more delay in the data transmission. Due to energy depletion of the intermediate nodes the routing protocol finds many alternate path to deliver the packets. Hence the conventional network has more delay compare to CL_EHSN.

\section{Conclusion and Future Work}

The basic idea behind our protocol is to re-energize the neighboring nodes using the RF energy transmitted by the active nodes along the routing path. This is achieved by designing new energy harvesting sensor node and 


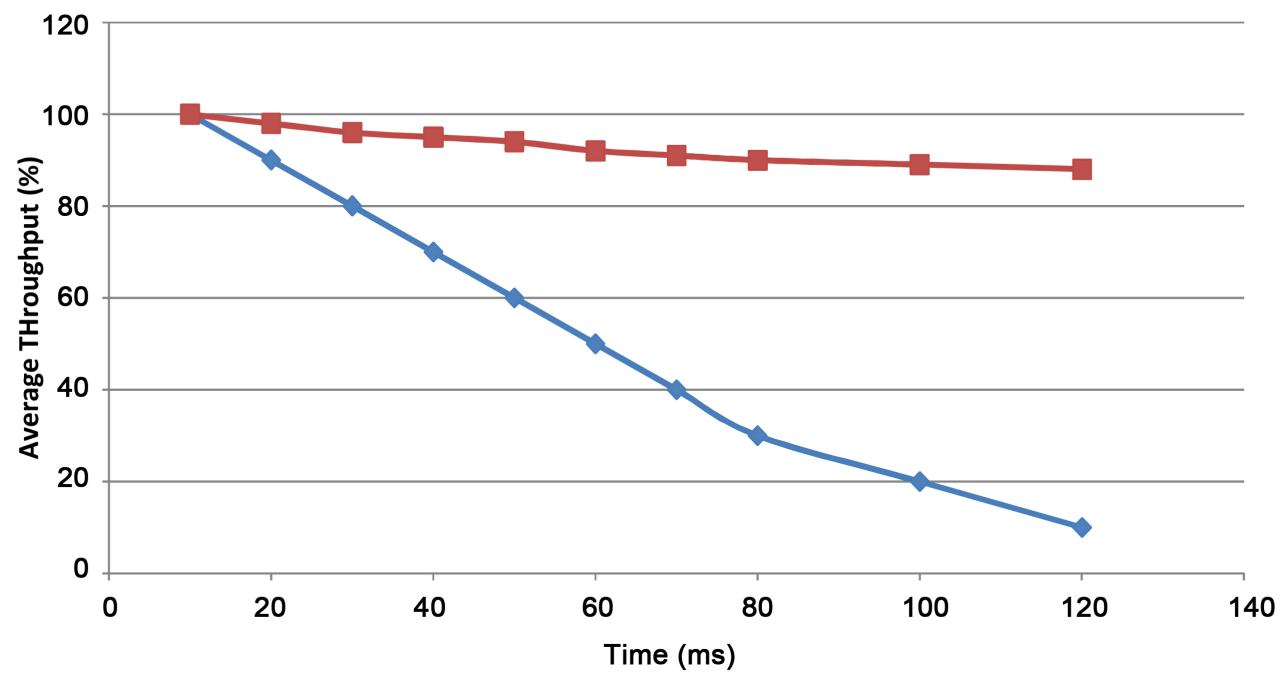

Figure 12. Average throughput of EHSN over simulation time.

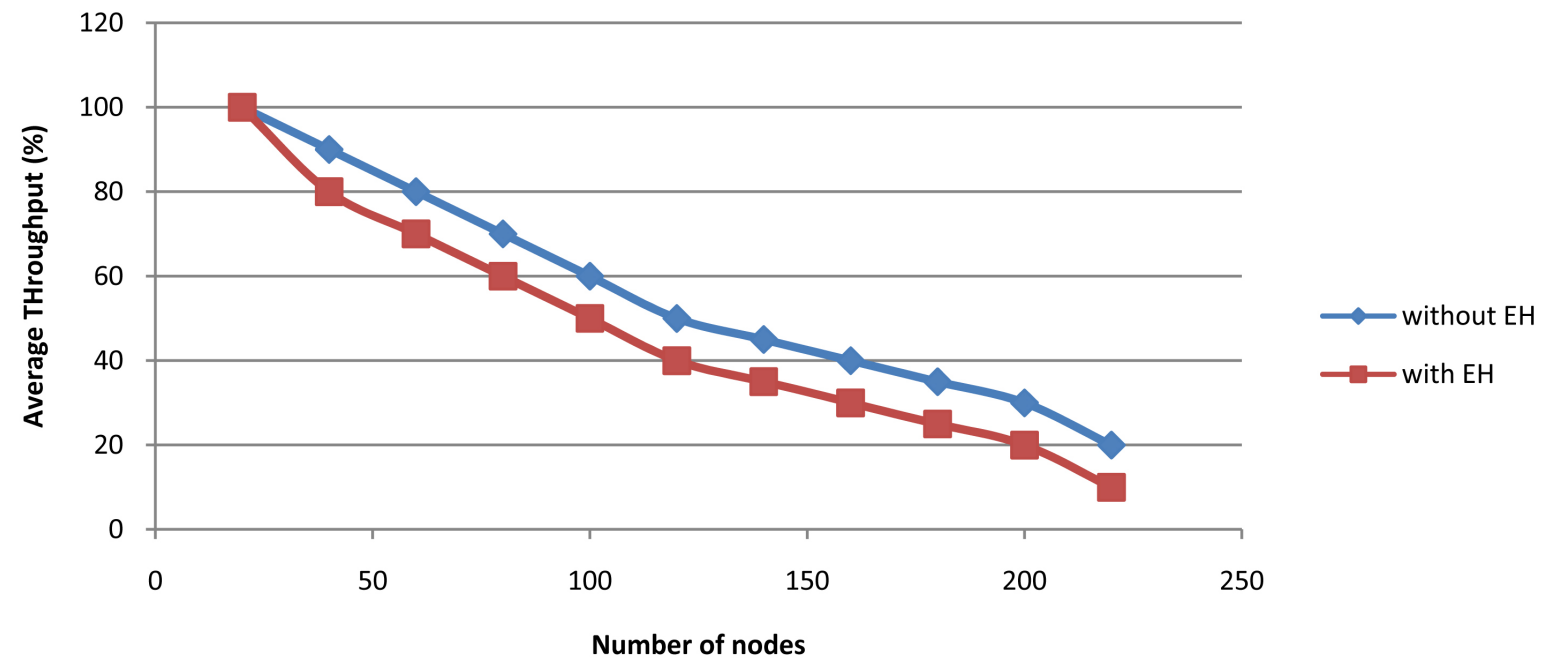

Figure 13. Performance of average network Throughput with respect to number of nodes in the network.

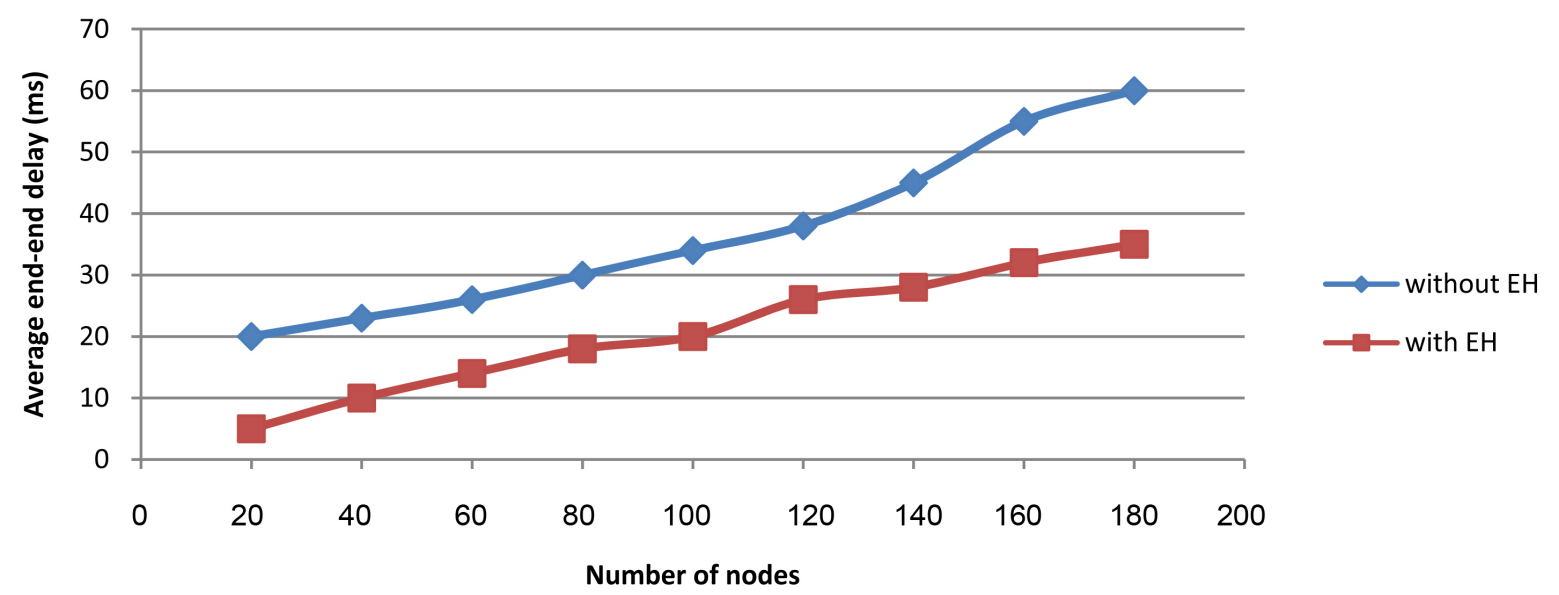

Figure 14. Performance of Average end-end delay with respect to number of nodes. 
redesigning the MAC protocol. The results are evaluated using real time experimental set up and it is proved that the lifetime of the sensor node is improved by $40 \%$. In future, the CL_EHSN can be extended to design novel antenna to harvest more power from the spurious signals available in the environment.

\section{References}

[1] Nintanavongsa, P., Muncuk, U., Lewis, D R. and Chowdhury, K.R. (2012) Design Optimization and Implementation for RF Energy Harvesting Circuits. IEEE Journal on Emerging and Selected Topics in Circuits and Systems, 2, 24-33. http://dx.doi.org/10.1109/JETCAS.2012.2187106

[2] Rahimi, M., Shah, H., Sukhatme, G.S., Heidemann, J. and Estrin, D. (2003) Studying the Feasibility of Energy Harvesting in a Mobile Sensor Network. IEEE International Conference on Robotics and Automation (ICRA), Taipei, 14 September 2003, 19-24. http://dx.doi.org/10.1109/robot.2003.1241567

[3] Paradiso, J.A. and Starner, T. (2005) Energy Scavenging for Mobile and Wireless Electronics. IEEE Pervasive Computing, 4, 18-27. http://dx.doi.org/10.1109/MPRV.2005.9

[4] Simjee, F.I. and Chou, P.H. (2008) Efficient Charging of Super Capacitors for Extended Lifetime of Wireless Sensor Nodes. IEEE Transactions on Power Electronics, 23, 1526-1536. http://dx.doi.org/10.1109/TPEL.2008.921078

[5] Dutta, P., Hui, J., Jeong, J., Kim, S., Sharp, C., Taneja, J., Tolle, G., Whitehouse, K. and Culler, D. (2006) Trio: Enabling Sustainable and Scalable Outdoor Wireless Sensor Network Deployments. IPSN, Nashville, 19-21 April $2006,407$. http://dx.doi.org/10.1145/1127777.1127839

[6] Sikka, P., Corke, P., Valencia, P., Crossman, C., Swain, D. and Bishop Hurley, G. (2006) Wireless Adhoc Sensor and Actuator Networks on the Farm. IPSN'06 Proceedings of the 5th International Conference on Information Processing in Sensor Networks, Newyork, 492-499. http://dx.doi.org/10.1145/1127777.1127852

[7] Corke, P., Valencia, P., Sikka, P., Wark, T. and Overs, L. (2007) Long-Duration Solar-Powered Wireless Sensor Networks. EmNets, Cork, 25-26 June 2007, 33-37. http://dx.doi.org/10.1145/1278972.1278980

[8] Arms, S.W., Galbreath, J.H., Townsend, C.P., Churchill, D.L., Corneau, B., Ketcham, R.P. and Phan, N. (2009) Energy Harvesting Wireless Sensors and Networked Timing Synchronization for Aircraft Structural Health Monitoring. Wireless VITAE.

[9] Alippi, C. and Galperti, C. (2008) An Adaptive System for Optimal Solar Energy Harvesting in Wireless Sensor Network Nodes. IEEE Transaction on Circuits and Systems, 55, 1742-1750. http://dx.doi.org/10.1109/TCSI.2008.922023

[10] Ammar, Y., Buhrig, A., Marzencki, M., Charlot, B., Basrour, S., Matou, K. and Renaudin, M. (2005) Wireless Sensor Network Node with Asynchronous Architecture and Vibration Harvesting Micro Power Generator. Proceedings of the 2005 Joint Conference on Smart Objects and Ambient Intelligence: Innovative Context-Aware Services: Usages and Technologies, Grenoble, 12-14 October 2005, 287-292. http://dx.doi.org/10.1145/1107548.1107618

[11] Vijayaraghavan, K. and Rajamani, R. (2007) Active Control Based Energy Harvesting for Battery-Less Wireless Traffic Sensors: Theory and Experiments. Proc. of American Control Conference, New York, 11-13 July 2007, 4579-4584. http://dx.doi.org/10.1109/acc.2007.4282842

[12] Mateu, L., Codrea, C., Lucas, N., Pollak, M. and Spies, P. (2006) Energy Harvesting for Wireless Communication Systems Using Thermo Generators. Proc. of the XXI Conference on Design of Circuits and Integrated Systems (DCIS), Barcelona, 22-24 November 2006, 22-24.

[13] Bottner, H., et al. (2004) New Thermoelectric Components Using Microsystem Technologies. Journal of Microelectromechanical Systems, 13, 414-420. http://dx.doi.org/10.1109/JMEMS.2004.828740

[14] Microstrain. http://www.microstrain.com

[15] Arms, S.W., Townsend, C.P., Churchill, D.L., Galbreath, J.H. and Mundell, S.W. (2005) Power Management for Energy Harvesting Wireless Sensors. Proc. of SPIE Intl Symposium on Smart Structures and Smart Materials, San Diego, 20 March 2005, 267-275. http://dx.doi.org/10.1117/12.600302

[16] Texas Instruments: http://www.ti.com

[17] Cerametricshttp: www.advancedcerametrics.com

[18] Penella, M.T. and Gasulla, M. (2007) A Review of Commercial Energy Harvesters for Autonomous Sensors. Proceeding of Instrumentation and Measurement Technology Conference (IMTC), Warsaw, 1-3 May 2007, 1-5. http://dx.doi.org/10.1109/imtc.2007.379234

[19] Ambiosystems: http://www.ambiosystems.com

[20] EnOcean: http://www.enocean.com

[21] Kartsakli, E., et al. (2014) A Survey on M2M Systems for mHealth: A Wireless Communications Perspective. Sensors, 14, 18009-18052. http://dx.doi.org/10.3390/s141018009 
[22] Esteves, V., Antonopoulos, A., Kartsakli, E., Puig-Vidal, M., Miribel-Català, P. and Verikoukis, C. (2015) Cooperative Energy Harvesting-Adaptive MAC Protocol for WBANs. Sensors, 15, 12635-12650. http://dx.doi.org/10.3390/s150612635

[23] Mekikis, P.V., Antonopoulos. A., Kartsakli, E., Lalos, A.S., Alonso, L. and Verikoukis. C. (2016) Information Exchange in Randomly Deployed Dense WSNs with Wireless Energy Harvesting Capabilities. IEEE Transactions on Wireless Communications, 15, 3008-3018. http://dx.doi.org/10.1109/TWC.2016.2514419

[24] Ibarra, E., Antonopoulos, A., Kartsakli, E. and Verikoukis, C. (2015) HEH-BMAC: Hybrid Polling MAC Protocol for Wireless Body Area Networks Operated by Human Energy Harvesting. Telecommunication Systems, 58, 111-124. http://dx.doi.org/10.1007/s11235-014-9898-z

[25] Mekikis, P.V., Lalos, A.S., Antonopoulos, A., Alonso, L. and Verikoukis, C. (2014) Wireless Energy Harvesting in Two-Way Network Coded Cooperative Communications: A Stochastic Approach for Large Scale Networks. IEEE Communications Letters, 18, 1011-1014. http://dx.doi.org/10.1109/LCOMM.2014.2320926

[26] Ibarra, E., Antonopoulos, A., Kartsakli, E., Rodrigues, J.J.P.C. and Verikoukis. C. (2016) QoS-Aware Energy Management in Body Sensor Nodes Powered by Human Energy Harvesting. IEEE Sensors Journal, 16, 542-549. http://dx.doi.org/10.1109/JSEN.2015.2483064

\section{Submit or recommend next manuscript to SCIRP and we will provide best service for you:}

Accepting pre-submission inquiries through Email, Facebook, LinkedIn, Twitter, etc.

A wide selection of journals (inclusive of 9 subjects, more than 200 journals)

Providing 24-hour high-quality service

User-friendly online submission system

Fair and swift peer-review system

Efficient typesetting and proofreading procedure

Display of the result of downloads and visits, as well as the number of cited articles

Maximum dissemination of your research work

Submit your manuscript at: http://papersubmission.scirp.org/ 